\title{
ROBOTIC APPLICATION IN NEUROSURGERY USING INTELLIGENT VISUAL AND HAPTIC INTERACTION
}

\author{
Jerbic, B. ; Nikolic, G. ${ }^{* *}$; Chudy, D. ${ }^{* * *}$; Svaco, M. ${ }^{*} \&$ Sekoranja, B. \\ *University of Zagreb, Faculty of Mechanical Engineering and Naval Architecture, Ivana Lučića 5, \\ Zagreb, Croatia \\ ${ }^{* *}$ University of Zagreb, Faculty of Textile Technology, Prilaz baruna Filipovića 28a, Zagreb, Croatia \\ ${ }^{* * *}$ Clinical Hospital Dubrava, Avenija Gojka Ššska 6, Zagreb, Croatia \\ E-Mail: bojan.jerbic@fsb.hr, gojko.nikolić@ttf.hr, darko.chudy@kbd.hr, marko.svaco@fsb.hr, \\ bojan.sekoranja@fsb.hr
}

\begin{abstract}
Today, the complexity and high technical requirements of neurosurgical operations are so demanding that modern robotic achievements and advances of accompanied technologies appear as the immanent means, which can significantly improve neurosurgical practice. A novel robotic system (RONNA RObotic NeuroNAvigation) for application in neurosurgery is presented. The RONNA consists of two conventional articulated robot arms with a total of 13 degrees of freedom. A rigid and accurate robot is used for precise targeting of planned operating points and a compliant robot is used as operative assistant. A distinctive marker was developed for the purpose of precise localization and registration of the patient's head. A novel visual calibration method is presented. The developed dual arm neurosurgical system enables flexible and reliable application with embedded behaviour based control providing intuitive interaction with surgical team and new possibilities compared to the existing surgical robot solutions.

(Received in March 2014, accepted in October 2014. This paper was with the authors 1 month for 1 revision.)
\end{abstract}

Key Words: Neurosurgery, Robotics, Preoperative Planning, RONNA

\section{INTRODUCTION}

Minimally invasive or less invasive neurosurgery nowadays is a common name for all fields of neurosurgery. However, stereotactic neurosurgery demands high accuracy during the procedure for reaching the target point in the intracranial space. The first attempts to create minimally invasive neurosurgery were made during the late 1940s and the early 1950s [1] with the development of stereotactic neurosurgery. In the early stage of stereotactic neurosurgery the imaging of the intracranial space was the most limiting factor. With the development of computed tomography (CT), intracranial tumours could be visualized which allowed stereotactic brain tumour biopsies. Magnetic resonance imaging (MRI) can show some neuroanatomical structures, like subthalamic or globus pallidus internus, relevant to movement disorder. With the use of MRI and CT we can define the position of active contact of the electrode for deep brain stimulation in postoperative monitoring [2]. The evolution of imaging technique with advances in computer technology enables the fusion of some sequences of MRI and tractography, which makes neurosurgery safer [3]. Modern neuroimaging techniques have not only a great impact on neuroradiology but they have also caused substantial improvements in all the fields of neurosurgery. However, during the evolution of neuroimaging the stereotactic frames were not changed dramatically; some changes were made in stereotactic localizers and use of non-ferromagnetic materials was employed. Modern stereotactic frames are sufficiently accurate in the present clinical practice. Human vision, with the capability of discerning $1 / 3$ of a millimetre, on average is the limiting factor in the stereotactic frame precision. 
The evolution of neuroimaging provides an insight into neuroanatomical structures, which in the past could be seen only on post-mortem stained brain tissue [4, 5]. Advances in technology have opened up new possibilities during preoperative planning, neurosurgical intervention, and intraoperative strategy. New hardware, which could substantially improve the accuracy of neurosurgical procedures, would help us to meet the near future more prepared. The hardware without hindrance to the necessary freedom of human creativity should be the most important factor during surgery. One of possible answers is a robotic system. Use of robots in neurosurgery is specific in comparison to other surgical applications. Since the operating target is not visible, the operation is feasible only through processing of the virtual image of patient's head acquired from appropriate kind of scan technology. Similar assumptions are made in the current clinical practice with the use of stereotactic methods. Robotic application in stereo-navigation ensures accuracy improvements, reliability and enables the surgeon to focus on the purpose and goals of the operation. Besides, bearing in mind the fact that modern artificial intelligence methods allow programming robots in a way that turns them into cognitive agents, robots applied in neurosurgery are not solely mechanical assistants but also intelligent participants. Thus, the robotic surgery has become an established part of clinical practice with numerous advantages $[6,7]$.

In this paper a dual arm robotic system (RONNA) for application in neurosurgery is presented. The RONNA consists of two conventional articulated robot arms with a total of 13 degrees of freedom. A short description of the preliminary system can be found in [8]. The preliminary system [8] examines different types of robot configurations and describes the first working prototype. This paper proposes improvements over the previously presented system. It introduces a new localization feature, an improved planning system and new robot behaviour control models. The paper is organized as follows. In Section 2 the state of the art in robotic neurosurgery is presented. Section 3 presents the main objectives and features of the proposed RONNA system. In Section 4 all the details regarding the RONNA system are presented. Section 5 gives an overall conclusion and highlights future work.

\section{STATE OF THE ART}

Robotic application is obviously a step ahead when compared to the conventional techniques. The most common one is a stereotactic frame, which is usually fixed to the patient's head when using an invasive method. Then, the patient is scanned by the CT or MRI technology and the points of interest and approach angles are determined using specific software. During the operation, the positions are calculated manually and then they are set on the frame head that now points to the desired target. The required tool or instrument can be mounted on the frame and positioned correctly. Other proposed techniques are imaging-guided surgery and robotic surgery. The former is used when there is no need for very precise interventions due to a higher degree of inaccuracy. The robot, on the other hand, is very precise. There are a few proposed and currently used robots in medical practice. The main differences between them are in the method of localization of the patient's head. One approach is the use of teleoperated robots where the surgeon controls the movement of the robot via some haptic interface and the robot replicates the surgeon's movement, but these solutions are not common in neurosurgery. The teleoperated techniques have the drawback of significantly prolonging the operation. Other methods, along with the one proposed in this article, use the robot localization marker instead of the frame. Currently there are a few commercially available solutions and more of them are in the research phase. Various localization methods are proposed. Frameless stereotactic robot Rosa is a surgical system released by Medtech [9, 10]. The system is intended for needle-based intracranial procedures. Rosa localization system is based on markerless registration. To localize the patient a scan of the patient head is 
performed, and the measured points are overlapped with ones located at radiology image. A different solution comes from Renishaw company - NeuroMate is a commercially available, image guided, robotic-assisted system [11, 12]. The robot can be used with a stereotactic frame or in frameless mode. For the frame-based procedures there is no need for registration because NeuroMate is already calibrated within the frame coordinate system. In frameless mode the localizing is based on a helicopter-shaped localizing device. The localizing device along with CT and MRI localizers is fixed to the patient with insertion to an implanted base. NeuroMate uses either infrared sensors or ultrasound as methods for capturing common point needed for registration. The third alternative product is Prosurgics PathFinder, a neurorobotic system that is mobile, with a very stable base that can be wheeled in and out of the operating room. PathFinder locates the surgical field by identifying reflectors attached to the patient's head, using a camera system integrated in its distal arm [13]. Fiducials are impregnated with radio-opaque material to be visible in $\mathrm{CT}$ and coated with reflective material so they can be registered with a camera. The fiducials are fixed to the skin using adhesive tape, or alternatively attached to a plate fixed to the skull. A different approach can be observed within a teleoperated neurosurgical robot neuroArm [14]. A specific MRI compatible robotic solution was developed and manufactured. The main focus of the presented research was to reproduce visual, audio and haptic feedback within a neurosurgery procedure at a remote workstation.

In comparison to other solutions RONNA uses a three spheres marker for patient localization placed near the point of interest. Mathematical principles dictate that the closer the reference frame is to the target point the precision is higher. Feature based contactless methods heavily rely on face features. As the rest of the head is practically featureless noninvasive feature dependent methods are more precise in point closer to the front of the patient head than in the rear which isn't the case with marker based methods. Main difference is that RONNA provides a dual-arm configuration with novelties particularly in the field of intelligent multi-robot control, contextual interpretation of the environment and intelligent interaction with humans, both in terms of functionality and safety. Also RONNA is able to perform precise bone drilling with continuous force feedback, which improves patient safety and minimizes potential trauma caused by manual drilling procedures.

\section{OBJECTIVES}

This work presents a specific innovative approach to the application of robots in neurosurgery. The developed localization method of a patient, which is a crucial problem in neurosurgical robotics, is based on contactless stereovision system and a localization feature attached to the patient's head. The vision system attached to the end of the robot arm provides not only registration of the patient's spatial position but also the self-calibration capability. Namely, the direct application of robotic technology in neurosurgery cannot fully satisfy the precision requirements. Unlike industrial applications where robots work in a structured environment, in neurosurgery robot must guide the movements towards positions (coordinates), which are gathered (processed) from CT or MR images whose coordinates are transformed to the current position of the patient in the robot coordinate system. This requires inverse kinematic calculation to determine the related robot joints positions. Since the robot is an open kinematic chain, even small unavoidable displacement errors in some joints, particularly those at the beginning of the chain, cause large position deviation of the end of the robotic arm. This problem can be solved with two approaches. One assumes the development of a special-purpose robot built from very precise components, which leads to high costs. Bearing in mind that in accordance with the laws of nature and technical constraints, it is not possible to produce absolutely correct components, nor control the system 
without deviation. Thus, the approach is predestined to limited success. This work relies on the second solution. Recognizing the natural uniqueness, imperfections and variance (like the humans are), there is no need to have perfect robots, but enough adaptable to the mentioned conditions. It assumes the development of the system based on commercial robots available on the market, but focusing the research on development of adaptive and intelligent control system, which can provide specific robot behaviour and adaptability to its own imperfection.

Special attention has been directed toward interaction between surgeon and robot. Using impedance control the surgeon can precisely guide the robot interactively, but in the restricted way according to the requirements of the operation plan and the anatomy of the patient head. Due to reliable precision of the system the operation could be done very accurately and faster than traditional techniques limiting the unwanted effects to the patient. With all the research in this field and further technology improvements and advances in the field of artificial intelligence robots will become even better assistants as they will also be able to anticipate and prepare themselves for certain phases and situations in the operating procedure.

\section{THE RONNA SYSTEM}

The RONNA system comprises navigation and planning software, two robots (master and assistant), a stereovision system, a localization feature (marker), operating tools (grippers, guides etc.) and advanced context-aware control software. In this paper, the accent is placed on the presentation of the basic principles of the developed system and does not describe in detail specifically developed scientific contributions, particularly in the field of intelligent multi-robot control, contextual interpretation of the environment and intelligent interaction with humans, both in terms of functionality and safety.

\subsection{Setup}

A dual arm robot setup was developed for robot assisted surgery as given in Fig. 1. The robots are standard units: one 6 degrees of freedom (DOF) robot (master robot) and one 7 DOF robot (assistant robot). The implementation of the standard robots ensures the robustness and costeffectiveness of the developed system. Master robot (KUKA KR6) is a revolute robot with a reach of $900 \mathrm{~mm}$, payload $6 \mathrm{~kg}$, and repeatability $\pm 0.03 \mathrm{~mm}$. This robot is used as the alternative to the common stereotactic method because of its stiffness and rigidity. The 7 DOF (Kuka LWR 4+) robot has a different architecture and can exhibit compliant behaviour. Assistant robot cannot demonstrate stiff behaviour and consequently has low absolute accuracy. Its drives are equipped with torque sensors, which provide soft interactive behaviour and adjust reactive motor torques through impedance control.
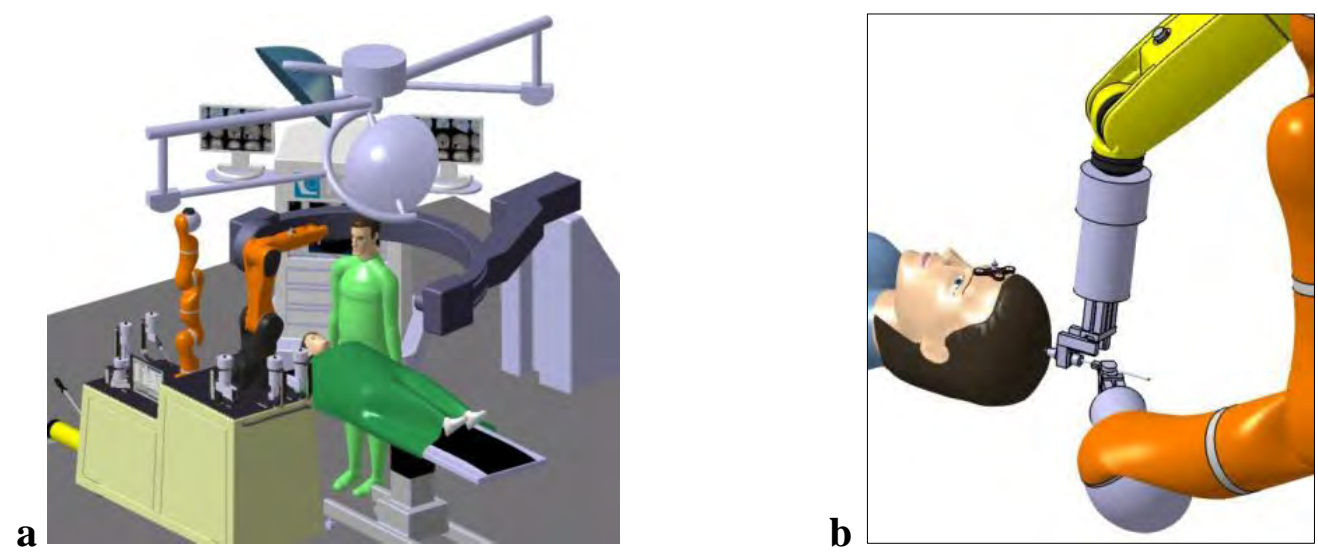

Figure 1: a) The RONNA system in operating room, b) the master robot used for accurate routing of the instruments. 
The robot can be programmed in a way that prevents the possibility of human injury or dangerous collisions with surrounding objects. The master robot can be used for accurate routing of the instruments towards the exact point of operation done by assistant robot or surgeon (Fig. 1 b). System design and functional requirements for the application in neurosurgery are much more demanding than in conventional robotics, such as industrial applications. First of all, the overall size of the system should be compact enough to fit in the operating room and to prevent interfering with the work of medical staff. On the other hand, the robotic system must meet complex requirements in terms of spatial working ability, using the longest possible reach of the robot arms. Therefore, the robot system setup was designed based on the application of CAD software modelling and simulations. Various neurosurgical operating trajectories and instruments were explored, as well as the requirements to accommodate the whole system in the operating room in relation to other equipment and personnel. The obtained results indicate the possibility of using different configurations: console and parallel (Fig. 2).
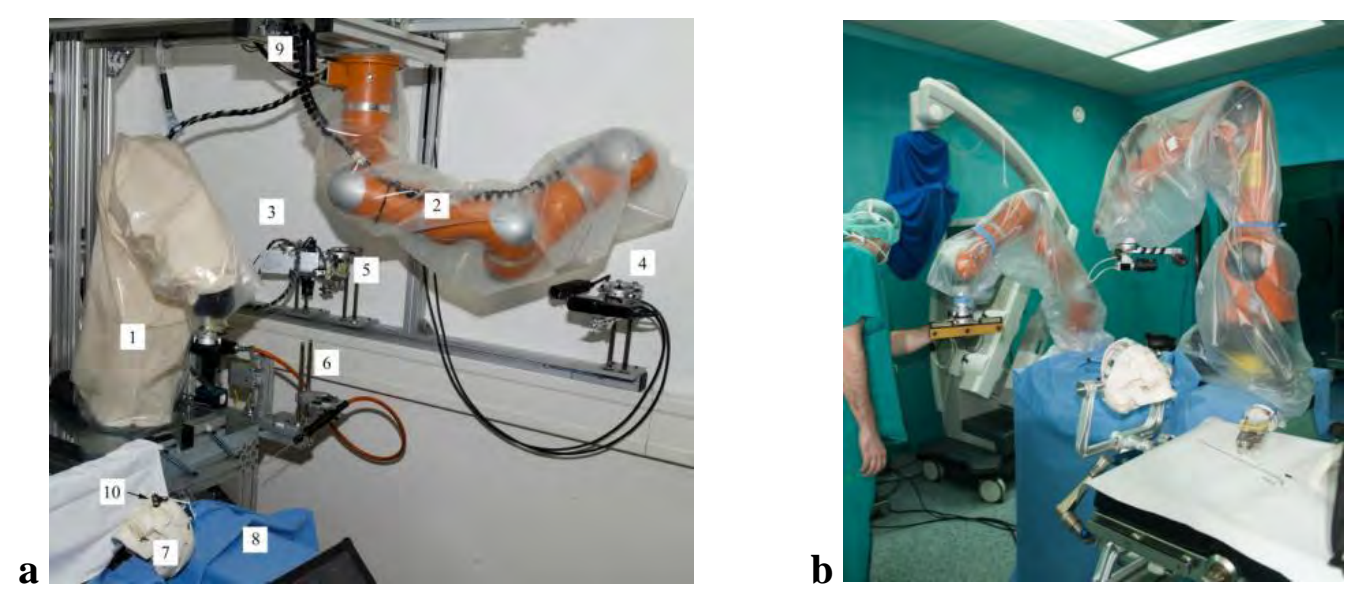

Figure 2: Two system configurations: console (a) and parallel (b); 1 - master robot, 2 - assistant robot, 3 and 4 - vision systems, 5 - instrument guide, 6 - drilling head, 7 - phantom head, 8 - operating table, 9 - ambient camera, 10 - marker.

Since RONNA provides a dual-arm configuration, a special control system based on the evolutionary algorithm to coordinate the movement of two robots developed in [15], is applied. The control algorithm is used to calculate offline trajectories in the planning and simulation phase. At this moment the algorithm cannot cope with dynamic changes in the environment. The algorithm is mainly used for preventing collision between the master and assistant robot by calculating their trajectories in two perpendicular planes. In the case where movements of surgical staff are detected in the robots workspace, the robots can either slow down their movement of pause execution.

\subsection{Operation planning and navigation}

The planning and navigation software RONNAplan is developed in MATLAB to help preparation of the robotic operation using CT or MR scans. The software is able to interpret DICOM standard image format from tomography scanners and provide $2 \mathrm{D}$ and $3 \mathrm{D}$ visualization (Fig. 3). The main purpose of the software is to define the approaching trajectory to the operation region in the brain through a cranial penetration by indicating related points. In order to define the trajectory the operating surgeon has to define two points - entry and target point [16]. Using mathematical calculation target and entry are connected with a line that intersects all related layers scanned, allowing the surgeon to verify and possibly modify the path. 


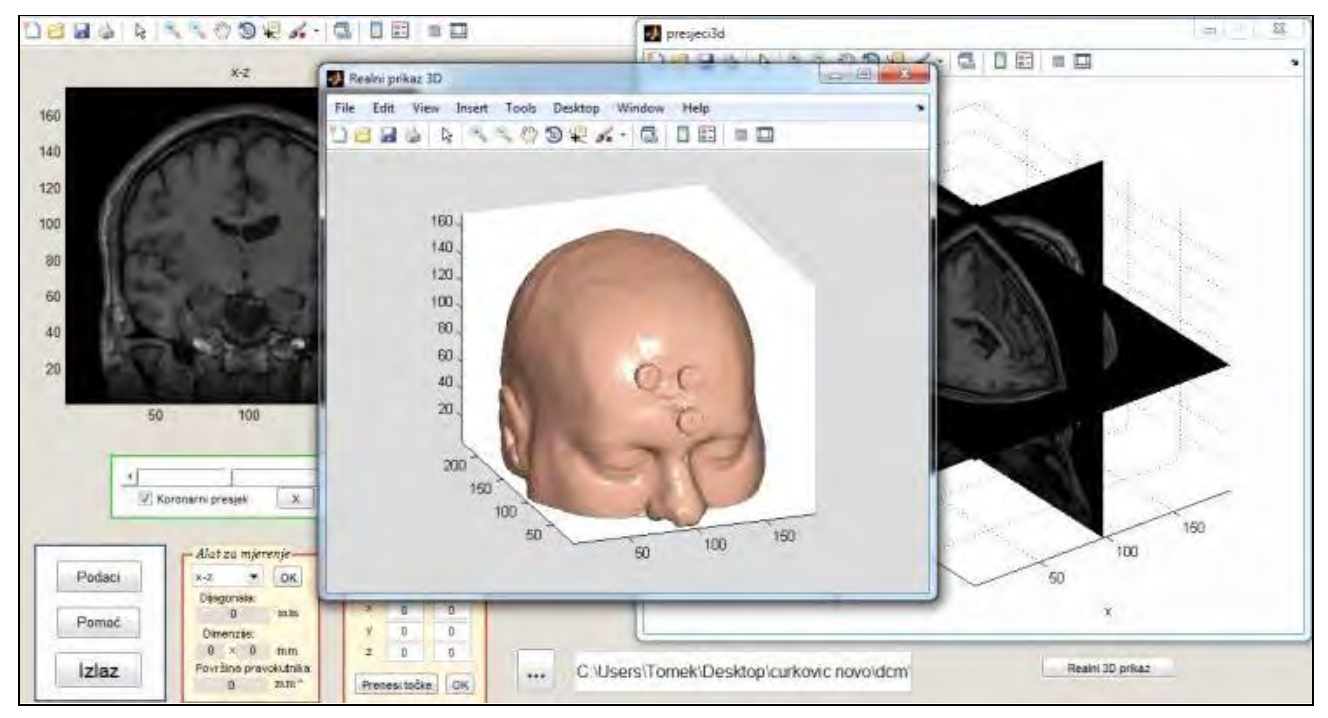

Figure 3: The planning and navigation software interface.

The verified points are then transferred to the robots. When RONNA finds the location of the patient's head by registering the information from CT or MR tomography the robotic procedure can begin. The surgeon can simultaneously monitor the progress using the RONNAplan software that is connected with robots and receives their position information, displaying the current position of the instrument on the scanned images.

\subsection{Localization feature}

A localization feature (marker) was developed to provide a means for localization and registration of the patient in operating room (Fig. 4). The marker attached to the patient's head enables the localization of the patient, along with identified operating points in the robot's coordinate system and consequently to transform the coordinate system of the scanner where the operating points are identified. Therefore the localization feature is a geometrical interface between the robots and CT or MRI scans. The basic accuracy of the RONNA system depends on the marker geometry, the vision system and scanning resolutions. The marker is designed to allow easy and minimally invasive fixing using just one dedicated bone screw. It consists of two parts: passive 3-spheres kit, mounting base with integrated bone screw. The interface between the 3-spheres kit and mounting base includes asymmetrical geometry features that allow unique positioning and easy mounting and dismounting. By using the stereovision system the centre point of each sphere can be determined. Three non-collinear points, which belong to the centres of the spheres, are sufficient to fully describe a body in 3D space thus forming the right-handed marker coordinate system $\mathbf{M}$. The screw is made from titanium alloy with a unique mating surface for the joining procedure with the marker. The attachment procedure is depicted in Fig. 4.
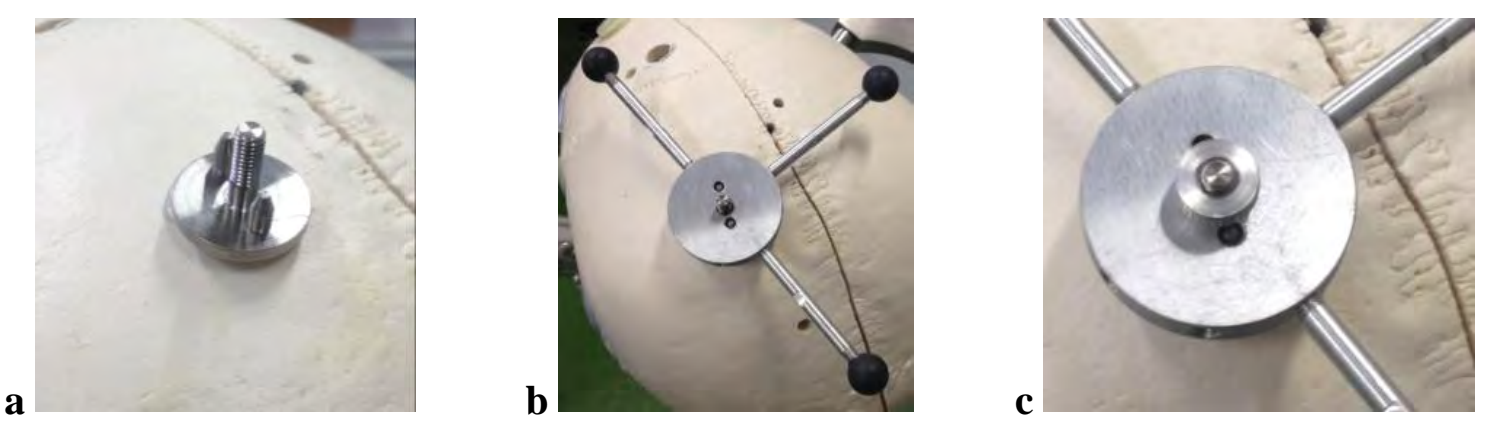

Figure 4: a) Attachment screw, b) and c) marker fastening procedure. 
Very important aspect of the localization feature design is mounting/dismounting repeatability, when the marker is detached and subsequently attached. Keeping only the titanium screw (with mount base) on the patient's head, during the pre-operation process, would be minor discomfort and the marker can be easily reattached to the screw. In order to test the accuracy and repeatability of the localization procedure the marker was fastened to a laboratory phantom. The marker was dismounted and reattached before each localization procedure. Measurements were performed to determine the measurement inaccuracy (repeatability) of the whole system, i.e. of the robot, reattachment procedure and vision system. The statistical analysis of the marker position and orientation (6D) performed on three samples each containing 30 measurements is given in Table I.

Table I: Localization accuracy of the marker reattached to the phantom head.

\begin{tabular}{|c|c|c|c|c|c|c|}
\hline Measurement & $x(\mathrm{~mm})$ & $y(\mathrm{~mm})$ & $z(\mathrm{~mm})$ & $\alpha\left(^{\circ}\right)$ & $\beta\left(^{\circ}\right)$ & $\gamma\left(^{\circ}\right)$ \\
\hline Mean Error & 0.022 & 0.001 & 0.002 & 0.002 & 0.004 & 0.011 \\
\hline Standard Deviation & 0.014 & 0.005 & 0.006 & 0.01 & 0.014 & 0.014 \\
\hline Maximum Error & 0.047 & 0.023 & 0.025 & 0.043 & 0.075 & 0.068 \\
\hline
\end{tabular}

By superimposing the spatial reproducibility error to a point located at a known distance (simulation of operation procedure) from the centre of the marker the terminal repositioning error is calculated. Table II represents coordinates in the marker coordinate system for six arbitrary points inside the skull (T0 - T6).

Table II: Simulated operation points located in RONNAmarker coordinate system.

\begin{tabular}{|c|c|c|c|c|c|c|}
\hline Operation point & $\mathrm{T} 0$ & $\mathrm{~T} 1$ & $\mathrm{~T} 2$ & $\mathrm{~T} 3$ & $\mathrm{~T} 4$ & $\mathrm{~T} 5$ \\
\hline $\mathrm{x}(\mathrm{mm})$ & 30 & 60 & 80 & 100 & 70 & 55 \\
\hline $\mathrm{y}(\mathrm{mm})$ & 35 & 60 & 60 & 50 & 70 & 40 \\
\hline $\mathrm{z}(\mathrm{mm})$ & 45 & 60 & 40 & 60 & 70 & 100 \\
\hline Euclidian distance $(\mathrm{mm})$ & 64.42 & 103.92 & 107.7 & 126.89 & 121.24 & 120.93 \\
\hline
\end{tabular}

Table III: Positioning error superimposed on operation points T0 - T6.

\begin{tabular}{|c|c|c|c|c|c|c|}
\hline Operation point & T0 & T1 & T2 & T3 & T4 & T5 \\
\hline Mean Error (mm) & 0.038 & 0.052 & 0.058 & 0.062 & 0.057 & 0.047 \\
\hline Standard Deviation (mm) & 0.038 & 0.052 & 0.053 & 0.057 & 0.058 & 0.054 \\
\hline Maximum Error (mm) & 0.146 & 0.209 & 0.215 & 0.241 & 0.237 & 0.232 \\
\hline
\end{tabular}

The operation points from Table II comprise a large portion of the area that will be delegated to the robot for neuronavigation. The Euclidian distance for all simulated operation points from marker origin varies from $64.42 \mathrm{~mm}$ to $126.89 \mathrm{~mm}$. Errors after marker repositioning, registered in Table I are superimposed on these points and the resulting Euclidian distances are given in Table III. The maximum repositioning error is $0.24 \mathrm{~mm}$ while the overall mean error is $0.035 \pm 0.037 \mathrm{~mm}$.

\subsection{Registration}

Using RONNAplan operating points and the centre points of the marker spheres, attached to the patient's head, need to be identified. Three points that belong to the marker define the coordinate system (frame) $\mathbf{M}$, which represents at the same time the coordinate system of the patient. The frame $\mathbf{M}$ (Fig. $5 \mathrm{~b}$ ) is calculated with origin in $\mathrm{P} 1$, direction of $\mathrm{X}$ axis from $\mathrm{P} 1$ to $\mathrm{P} 2$ and with point $\mathrm{P} 3$, subsequently defining the XY plane. Therefore, all coordinates of the operating points need to be transformed within RONNA planning software relatively to the frame M. In other words, after the operating plan is created all positions $(x, y, z)$ and 
orientations $(\alpha, \beta, \gamma)$ of the operating points and the surgical instruments are registered in the marker coordinate system (Fig. 5 b). Any other point of interest can be subsequently registered in the marker coordinate system. A fusion of the CT scan and an additional MRI scan can be done to ensure the visibility of all anatomical structures in the final image. A coordinate system CF denotes the frame that belongs to the CT scan, actually to the related images. Three points of the marker spheres, which define frame M (Fig. 5 b), are localized in the $\mathbf{C F}$ frame. Euler angles $\varphi, \theta, \psi$ and the corresponding translations $x_{M}, y_{M}, z_{M}$ defining the frame $\mathbf{M}$ with respect to $\mathrm{CF}$ are calculated.
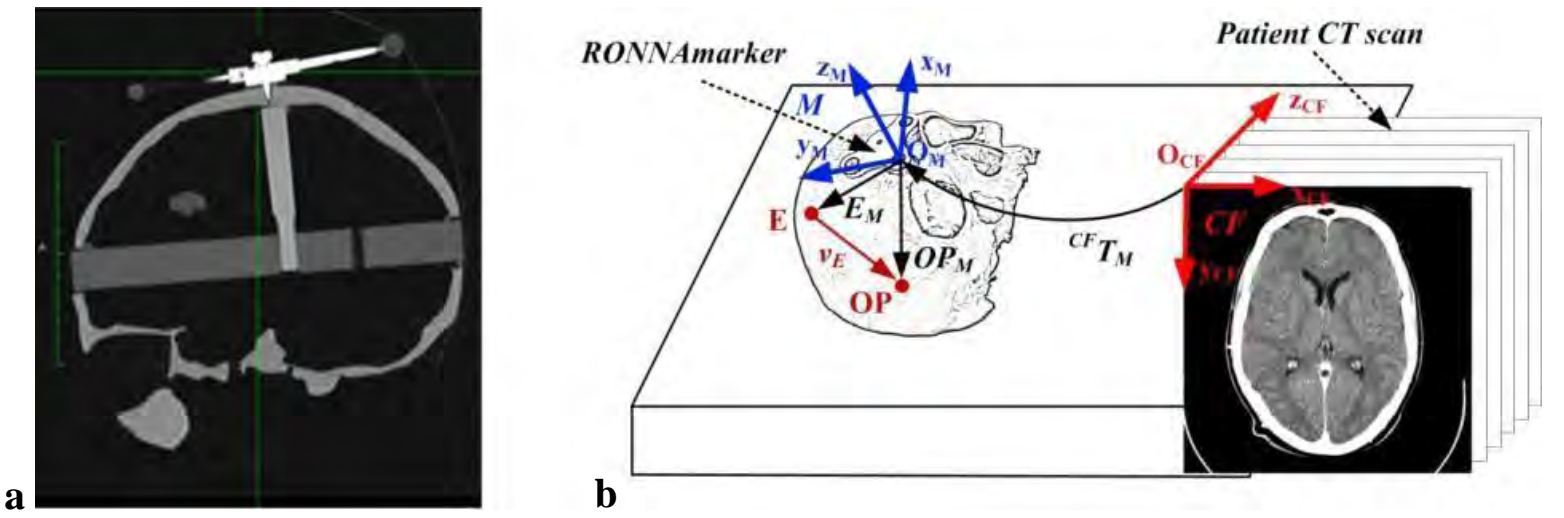

Figure 5: a) CT scan of the phantom with attached marker, b) RONNA coordinate frames.

The operating point op and the entry point $\mathbf{e}$ are localized in $\mathbf{C F}$, defining the entry vector $\mathbf{v}_{E}$. In order to define any point of interest in frame $\mathbf{M}$ typical homogenous transformation matrices $\mathbf{T}$ and $\mathbf{R}_{z y x}$ are applied [17, 18]. The terminal homogeneous transformation ${ }^{C F} \mathbf{T}_{M}$ is calculated as ${ }^{C F} \mathbf{T}_{M}=\mathbf{T} \cdot \mathbf{R}_{z y x}(\varphi, \theta, \psi)$. Operating point $\mathbf{o p}_{M}$ given in coordinate system $\mathbf{M}$ is calculated as $\mathbf{o p}_{M}=\mathbf{o p}_{C F}{ }^{C F} \mathbf{T}_{M}$, where the entry point is calculated as $\mathbf{e}_{M}=\mathbf{e}_{C F}{ }^{C F} \mathbf{T}_{M}$. The operation vector $\mathbf{v}_{E}$, i.e. the defined robot trajectory is given by $\mathbf{v}_{E}=\mathbf{v}_{E}\left(\mathbf{o p}_{M}, \mathbf{e}_{M}\right)$.

\subsection{Localization by vision system}

After the coordinate frames are registered in the patient's coordinate system the planning software is ready to send the information to the robots. In order to work with the operating points the robot must identify the position and orientation of the marker spheres that define the coordinate system of the patient. The localization process of the patient in the operating room is divided into two phases: approximate and final localization.

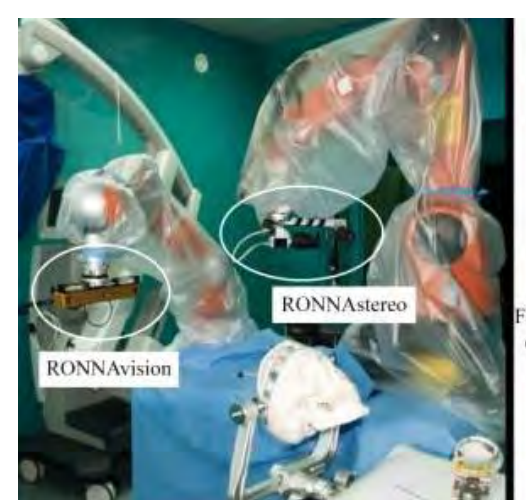

a

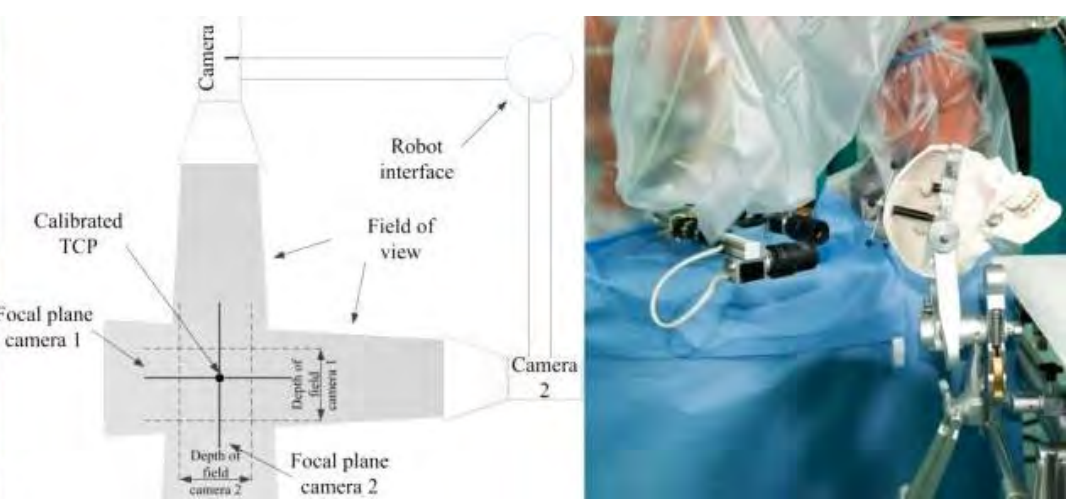

b

Figure 6: a) RONNAvision and RONNAstereo, b) RONNAstereo localization.

The first phase in the localization process is done with the RONNAvision module as shown in Fig. 6 a. The RONNAvision module is a standard stereovision camera that is 
mounted on the assistant robot. The robot localizes the marker attached to the patient head and sends the position information to the master robot. In the second phase of localization the master robot uses a specially designed stereovision system. This stereo-vision module (RONNAstereo) comprises two high resolution cameras with a very narrow field of view as shown in Fig. 6 b. The cameras are set up at right angle and the focus point of both cameras is in the intersection of their optical axes.

\subsection{Master robot calibration and precise error correction}

Despite the precise localization performed as described in the previous section, the significant deviations are immanent to the robotic technology. It comes from the summation of errors in an open kinematic chain of the robot, inevitable inaccuracies in the design of tools, instruments and tool changer itself. In order to provide sufficient accuracy, when the patient has been localized the master robot should recalibrate itself. Recalibration needs to be done according to planned working configurations. A working configuration is specific formation of robot links when robot approaches the planned working trajectory. For each different planned configuration the robot localizes the RONNAmarker and adjusts offsets for operating points which use this configuration. By this means the error produced by the open kinematic chain is reduced. Master robot uses a special tool guide for routing the surgical instruments like needle, drill or any other instrument to the planned position and desired orientation. The guide also allows the surgeon to be in direct contact with the patient, not allowing the robot full control over the operation procedure.

One other very practical means of error minimization used in the RONNA system is the fact that the virtual tool centre point of the RONNAvision module is actually the physical tip of the operating instrument. In this way every point localized by the RONNA vision is also an actual point that can be directly reached by inserting the operating instrument through the tool guide. Implementing these two principles of error minimization and correction the robot is able to provide satisfactory accuracy. To test the accuracy several experiments were performed in a laboratory environment on a phantom (polymer replica of a human skull). The phantom contains measurement pins attached to the top of the replica. The non-target end of the pin is secured to the plate forming a rigid connection. The target end of the pin is conically tapered to a point. Nine of these pins are distributed on the plate simulating points of interest identified by the surgeon. Two lines $1.5 \mathrm{~mm}$ apart are engraved on each rod, representing a reference scale in optical measurements of the vision system. Marker is attached to the phantom forehead to allow the localization of the phantom and thus the registration of the pin tips in the robot coordinates. When the plate is attached, the phantom is processed on a CT scanner. The images are interpreted and the coordinates of the pin tips are registered in the marker coordinate system. The positioning error of the robot system is measured using a vision system. The tool attached to the robot arm end effector is positioned at the simulated operating point (tip of the pin). Then the distance between the achieved position and the ideal position is measured. From two perpendicular projections, the vision system measures offset errors in all three directions $-\mathrm{x}, \mathrm{y}$ and $\mathrm{z}$. By using the precise reference engravings on the pins, the offset values in pixels are transformed into real values in millimetres and the error is calculated as Euclidian distance. Repeating the process with the phantom randomly placed on the operating table ensures a large measurement sample from which accuracy can be calculated. Accuracy was tested in the area close to the localization plate (Euclidian distance less than $100 \mathrm{~mm}$ ). The average error of all measurements was $0.36 \mathrm{~mm}$ with a standard deviation of $0.17 \mathrm{~mm}$ and maximum error of $0.71 \mathrm{~mm}$ which is comparable to stereotactic accuracy. 


\subsection{Application}

The RONNA system is designed to work in interactive assisted or autonomous mode, depending on the type of surgery and the surgeon choice. In both cases the patient should be in anaesthesia with the head fixed in Mayfield clamp. When RONNA works interactively just assisting the surgeon, the assistant robot has the assignment to approximately locate the patient by its stereovision system (Fig. 6 b). This will help the master robot to find the marker spheres attached to the patient's head. After localization and calibration procedures the master robot releases the cameras ready for operation. As given in Fig. 7 the interactive robot assisted application provides precise navigation of the instruments into direction of the planned point in cranial space, enabling surgeon to interactively adjust the guide (its distance or position) at the end of the robot arm. Wherever surgeon set the guide, it always points at the planned target of interest. The robot uses force and torque sensor to interpret the surgeon haptic intentions. In this scenario the robot replaces Leksell stereotactic frame, providing significantly easier, more versatile and faster operation.
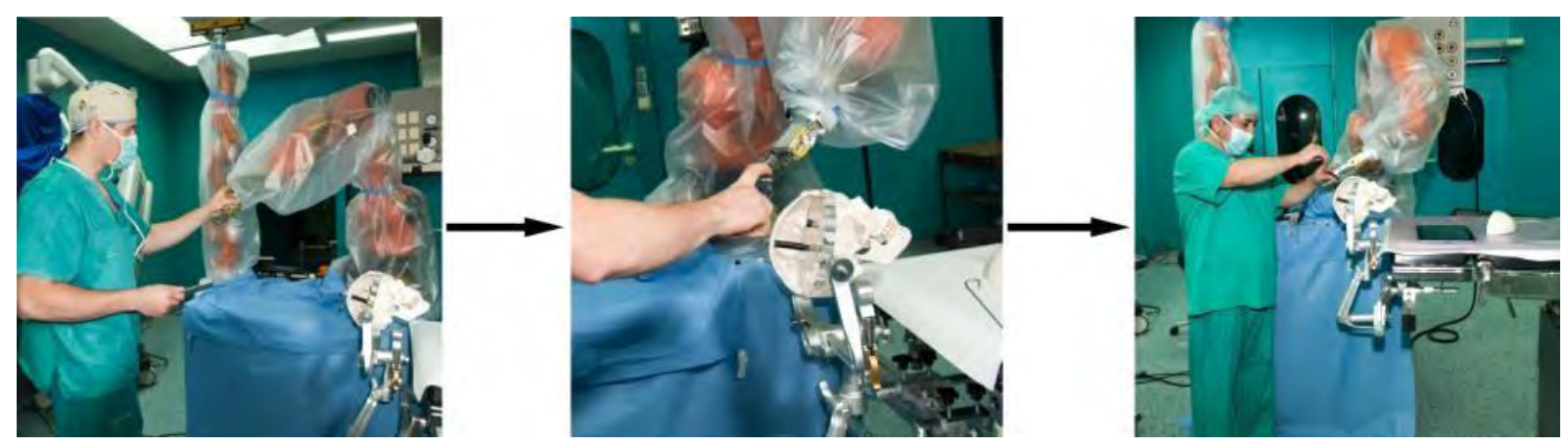

Figure 7: Interactive application during preclinical testing.

In the case when RONNA works in the autonomous mode both arms are active, functioning as coordinated dual arm system. The master robot positions the tool guide to the appropriate position and orientation. The assistant robot inserts the operating instrument through the tool guide pointing toward the point of operation (Fig. 8).

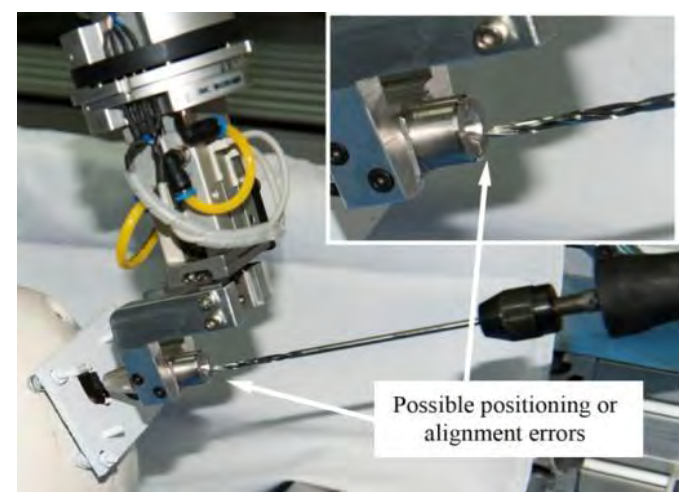

Figure 8: Expected positioning or alignment error by assistant robot.

RONNA control system includes all haptic capabilities based on force and torque sensors on the master robot and impedance control embedded in the assistant robot KUKA LWR4+. This provides flexible autonomous error correction of the assistant robot, adjusting itself to the precise tool guide provided by master robot. Besides, 7-axes torque sensitivity enables unparalleled bone drilling precision. The assistant robot driven by specially designed software leads the drilling head with exceptional sensitivity, stopping the drilling tool when the skull is penetrated. 


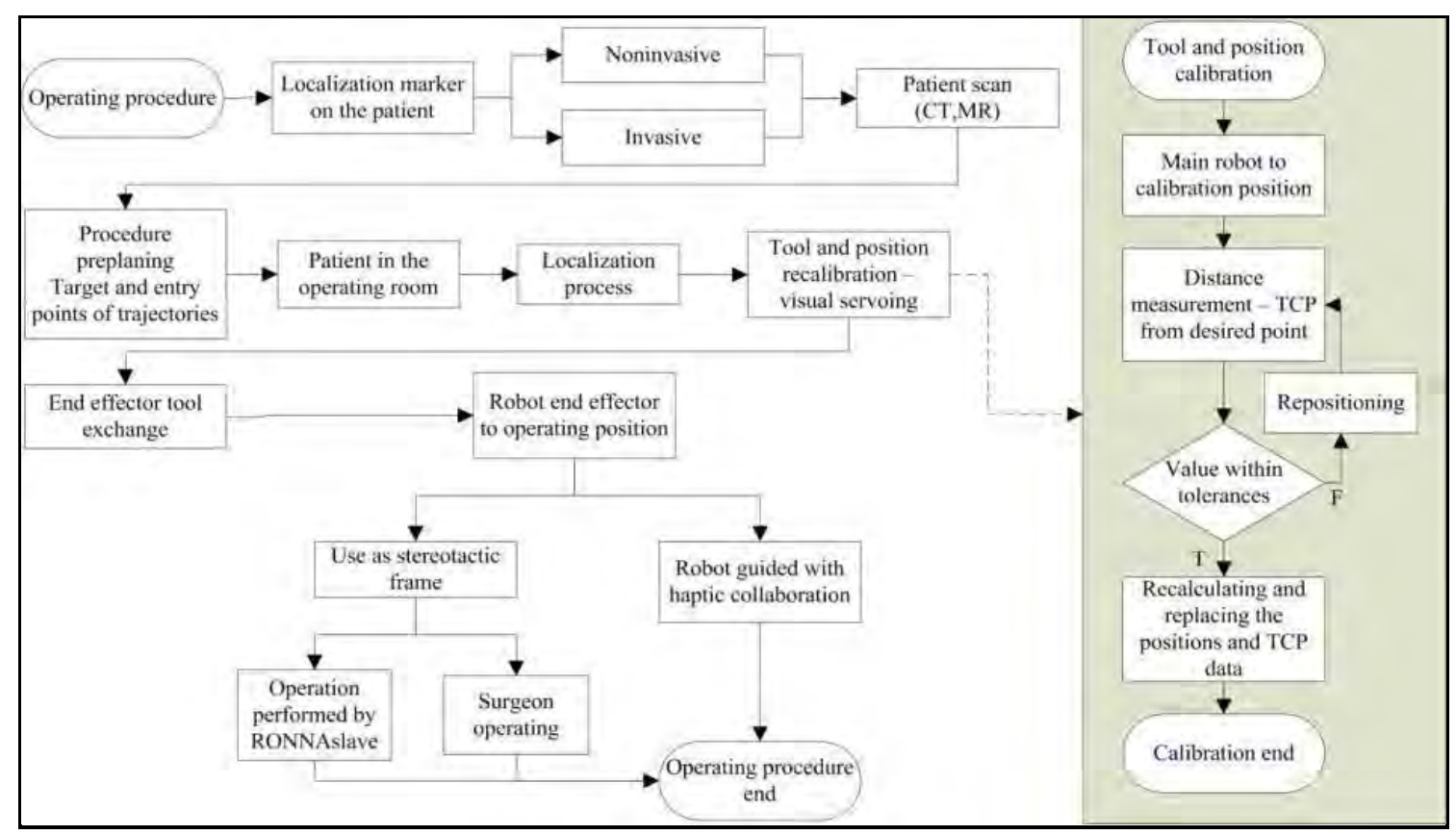

Figure 9: Operating procedure flowchart.

A detailed procedure for external ventricular drain (EVD) utilizing the RONNA system is described. An EVD is a temporary system which allows drainage of cerebral spinal fluid from the ventricles to an external closed system. In the first step a neurosurgeon mounts the RONNAmarker kit on a previously allocated portion of the patient head. The patient is then scanned on a CT or MRI device and the images are loaded in the RONNAplan module. The surgical team plans the entry and target points and validate the operation trajectory optimizing its length while avoiding functional areas within the brain. The patient, lying on a mobile operating table, is brought into the operating room (OR) and is anaesthetised. Patient head is

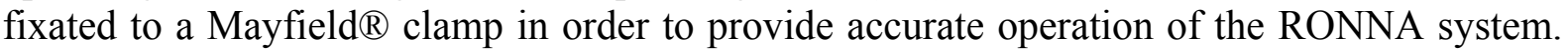
The RONNA system is positioned at a right angle to the patient. This position provides maximum manoeuvrability of both the robot and the surgeon while keeping the RONNA system at periphery when not in use. The robot is attached to the operating table using a rigid fixing arm. When all components are in place the robot controllers are turned on. The previously mentioned two step (coarse and fine) localization procedure is initialized for registration of robots in the marker coordinate system M. After the registration process is finished, the marker is detached from the patient head. The registration tools RONNAvision and RONNAstereo are removed and sterile covers are put on both robot arms. Appropriate tool guides, previously sterilised in plasma, are mounted on the system. First step of the operating procedure includes manual skin perforation at entry point indicated by the master robot. After the incision has been made the master robot positions the tool guide at the entry point. Either manual drilling or drilling by assistant robot is initiated with a bore of $3.2 \mathrm{~mm}$ in diameter. The dura is cauterized with a monopolar electrode (DIXI medical, France). A cerebral drainage catheter is inserted either manually of by the assistant robot. After successful insertion of the catheter, an elastic metal shaft is removed from the catheter bore. The master and assistant robots move to a predetermined position near the mobile platform base not to obscure the remaining procedure. The medical staffs fasten the catheter to the patient head and cover the entry area with sterile adhesive foil. With this step the operating procedure is finished. A post operational CT or MRI scan is made to validate proper catheter placement. The whole operating procedure has a predefined workflow as described above. Each of the robots, the assistant and the master robot, has predefined assignments. The robots are controlled by dedicated controlling units. For certain parts of the procedure intermittent 
motion of the robots is needed, for example in the drilling procedure. The intermittent motions are needed because they provide a more secure procedure, what is of primary importance to the surgical team and the potential patient.

\subsection{Intelligent control models}

The application of robots in the human environment assumes ability to work in unstructured environment and intuitive ability to interact with people, which is in contrast to conventional robot control methods [19]. Work in unstructured environment (operating room), means that the corresponding objects are not pre-defined, or not fully defined. Therefore, the robot cannot be programmed implicitly, since working conditions are not known, at least not all of them (position and behaviour of medical staff, location of other equipment etc.). Thus, robot behaviour and interaction with the environment must be based on the perception of the surrounding objects and the people who work with the robot. Perception must be spatial, i.e. in all three dimensions. Behaviour must be intuitive to liberate the surgeon dealing with the technical aspects of applied system. In RONNA system the dual stereovision system is applied to allow depth perception of the environment, not just the perception of image objects in a scene, but their 3D spatial relationship (Fig. 10).

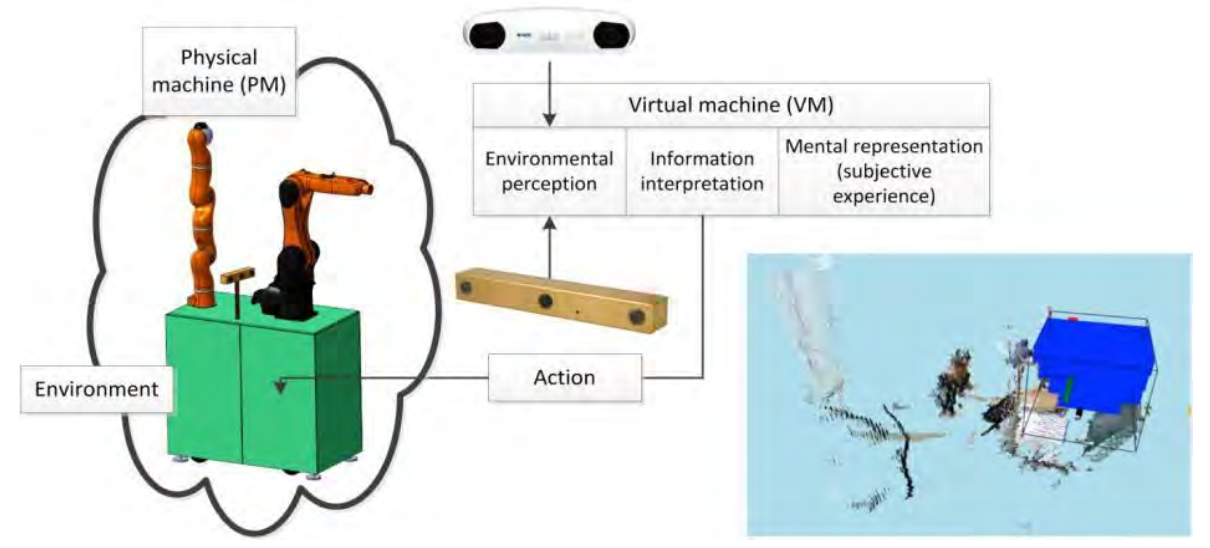

Figure 10: RONNA builds 3D interpretation of the surrounding world.

According to this RONNA is controlled in an "intelligent" way, adjusting the robots behaviour with regard to current situation or operating phase. Combining the control algorithms with principles of probabilistic reasoning and contextual understanding, developed in [20], RONNA is able to exhibit smart behaviour: bypassing obstacles, moving away from the staff when it is expected, preparing for expected actions based on perception. The mentioned advanced control solutions do not only contribute to a simple and reliable application of the system, but also increase safety that is an inevitable issue in such systems.

\section{CONCLUSION AND FUTURE WORK}

This article presents a new application of robotics in neurosurgery and a new localization method based on a dual-arm configuration with coordinated navigation. This research focuses on the use of robots instead of the usual stereotactic methods. With the introduction of new technologies in medical practice the RONNA system provides numerous advantages. The main advantage is a better and faster surgical procedure, which is also less invasive. Main contributions of the developed RONNA system can be observed through: novel interaction models for robots in the operating room, a novel patient localization method, specific tools for robotic applications in neurosurgery, new procedures in neurosurgical practice based on the application of robots, sensitive drilling with force feedback. Advanced control mechanisms 
using visual guidance, haptic feedback and context awareness algorithms allow an intuitive and natural interaction with the developed robotic system. Another significant feature of the RONNA system is the developed virtual operating environment (Fig. 1). It allows the surgical team precise preoperative planning where a detailed accommodation schedule for the RONNA system is specified. The complete operating procedure can be simulated in order to confirm practicability of the RONNA system for various robot assisted neurosurgical procedures. The presented system was extensively tested for the repeatability of the localization plate repositioning. The conducted measurements validate the system accuracy and consistency well within the desired precision specification. Redesign of tools attached to the end effector to bring the tool centre point closer to the flange will reduce the kinematic chain multiplication parameters, which will decrease the overall error.

RONNA system also introduces multiple robot configurations - single/multi arm, making the system even more flexible. After the preoperative phase, the RONNA system is capable of fully autonomous drilling, electrode implantation and catheter insertion. The system has the potential for applications in SEEG, biopsies, deep brain stimulation (DBS), tumour resection, and treating trigeminal neuralgia. Furthermore, the dual arm robot configuration has certain benefits compared to existing commercially available robot neuronavigation systems. The coupled dual-arm operation mode ensures higher stability and rigidity of the robot kinematic chains. The kinematic structure of the dual arm configuration in operations such as drilling, electrode and catheter insertion has only one degree of freedom, consequently providing better accuracy. For the distributed multiagent architecture, communication and collaboration algorithms have been implemented from earlier studies [21, 22].

The next step after validating the repeatability of the localization feature will include an extensive accuracy validation of the RONNA system on phantoms what is the usual practice [13] in neurosurgical robotics. Further laboratory and preclinical tests should prove that the developed system can advance neurosurgical procedures and considerably reduce time of operation. There are various direct or assisted surgical procedures planned with the RONNA system that will be presented where the main factor will be navigation accuracy around the target point which should be in rang as current neurosurgical practice or better.

\section{ACKNOWLEDGEMENTS}

Authors would like to acknowledge the Croatian Institute of Technology, Croatian Ministry of Science, Education and Sports for funding the project grant TP-11/0120-56: Robotic application in neurosurgery and Croatian Scientific Foundation through funding the research project $A C R O N-A$ new concept of Applied Cognitive RObotics in clinical Neuroscience. Authors would also like to acknowledge the support of the Faculty of Mechanical Engineering and Naval Architecture, University of Zagreb; Clinical Hospital Dubrava, Zagreb; Croatian Institute for Brain Research, School of Medicine, University of Zagreb.

\section{REFERENCES}

[1] Gildenberg, P. L. (2003). History repeats itself, Stereotactic and Functional Neurosurgery, Vol. 80, No. 1-4, 61-75, doi:10.1159/000075162

[2] Thani, N. B.; Bala, A.; Swann, G. B.; Lind, C. R. P. (2011). Accuracy of postoperative computed tomography and magnetic resonance image fusion for assessing deep brain stimulation electrodes, Neurosurgery, Vol. 69, No. 1, 207-214, doi:10.1227/NEU.0b013e318218c7ae

[3] Castellano, A.; Bello, L.; Michelozzi, C.; Gallucci, M.; Fava, E.; Iadanza, A.; Riva, M.; Casaceli, G.; Falini, A. (2012). Role of diffusion tensor magnetic resonance tractography in predicting the extent of resection in glioma surgery, Neuro-Oncology, Vol. 14, No. 2, 192-202, doi:10.1093/ neuonc/nor188 
[4] Yamada, K.; Akazawa, K.; Yuen, S.; Goto, M.; Matsushima, S.; Takahata, A.; Nakagawa, M.; Mineura, K.; Nishimura, T. (2010). MR imaging of ventral thalamic nuclei, AJNR, American Journal of Neuroradiology, Vol. 31, No. 4, 732-735, doi:10.3174/ajnr.A1870

[5] Kincses, Z. T.; Szabó, N.; Valálik, I.; Kopniczky, Z.; Dézsi, L.; Klivényi, P.; Jenkinson, M.; Király, A.; Babos, M.; Vörös, E.; Barzó, P.; Vécsei, L. (2012). Target identification for stereotactic thalamotomy using diffusion tractography, PLoS ONE, Vol. 7, No. 1, e29969, doi:10.1371/journal.pone.0029969

[6] Rosen, J.; Hannaford, B.; Satava, R. M. (2011). Surgical robotics - Systems applications and visions, Springer, New York

[7] McBeth, P. B.; Louw, D. F.; Rizun, P. R.; Sutherland, G. R. (2004). Robotics in neurosurgery, The American Journal of Surgery, Vol. 188, No. 4, 68-75, doi:10.1016/j.amjsurg.2004.08.004

[8] Nikolic, G.; Jerbic, B.; Chudy, D. (2013). Robotic applications in surgery with special emphasis on applications in neurosurgery, Katalinic, B.; Tekic, Z. (eds.), DAAAM International Scientific Book 2013, DAAAM International Vienna, 149-172, doi:10.2507/daaam.scibook.2013.06

[9] Dorfmüller, G.; Bulteau, C.; Fohlen, M.; Jalin, C.; Delalande, O. (2006). Frameless stereotactic robot-guided placement of depth electrodes for stereoelectroencephalography in presurgical assessment of children with refractory partial epilepsy, Abstracts of the $7^{\text {th }}$ European Congress on Epileptology, Helsinki, 1-2

[10] Gonzalez-Martinez, J.; Vadera, S.; Mullin, J.; Enatsu, R.; Alexopoulos, A. V.; Patwardhan, R.; Bingaman, W.; Najm, I. (2014.) Robot-assisted stereotactic laser ablation in medically intractable epilepsy: operative technique, Neurosurgery, Vol. 10, No. 2, 167-173, doi:10.1227/NEU. 0000000000000286

[11] Li, Q. H.; Zamorano, L.; Pandya, A.; Perez, R.; Gong, J.; Diaz, F. (2002). The application accuracy of the NeuroMate robot - A quantitative comparison with frameless and frame-based surgical localization systems, Computer Aided Surgery, Vol. 7, No. 2, 90-98, doi:10.1002/ igs. 10035

[12] Barua, N. U.; Lowis, S. P.; Woolley, M.; O’Sullivan, S.; Harrison, R.; Gill, S. S. (2013). Robotguided convection-enhanced delivery of carboplatin for advanced brainstem glioma, Acta Neurochirurgica, Vol. 155, No. 8, 1459-1465, doi:10.1007/s00701-013-1700-6

[13] Eljamel, M. S. (2007). Validation of the PathFinder ${ }^{\mathrm{TM}}$ neurosurgical robot using a phantom, The International Journal of Medical Robotics and Computer Assisted Surgery, Vol. 3, No. 4, $372-$ 377, doi: $10.1002 /$ rcs. 153

[14] Sutherland, G. R.; Wolfsberger, S.; Lama, S.; Zarei-nia, K. (2013). The evolution of neuroArm, Neurosurgery, Vol. 72, No. S1 (Supplement), A27-A32, doi:10.1227/NEU.0b013e318270da19

[15] Curkovic, P.; Jerbic, B.; Stipancic, T. (2013). Coordination of robots with overlapping workspaces based on motion co-evolution, International Journal of Simulation Modelling, Vol. 12, No. 1, 27-38, doi:10.2507/IJSIMM12(1)3.222

[16] Lumenta, C. B.; Di Rocco, C.; Haase, J.; Mooij, J. J. A. (2009). Neurosurgery, Springer Science \& Business Media, Berlin

[17] Krzic, P.; Pusavec, F.; Kopac, J. (2013). Kinematic constraints and offline programming in robotic machining applications, Technical Gazette, Vol. 20, No. 1, 117-124

[18] Dupac, M.; Noroozi, S. (2014). Dynamic modeling and simulation of a rotating single link flexible robotic manipulator subject to quick stops, Strojniski vestnik - Journal of Mechanical Engineering, Vol. 60, No. 7-8, 475-482, doi:10.5545/sv-jme.2013.1544

[19] Ferenc, G.; Dimic, Z.; Lutovac, M.; Vidakovic, J.; Kvrgic, V. (2013). Open architecture platforms for the control of robotic systems and a proposed reference architecture model, Transactions of FAMENA, Vol. 37, No. 1, 89-100

[20] Stipancic, T.; Jerbic, B.; Curkovic, P. (2012). Context-aware system applied in industrial assembly environment, International Journal of Advanced Robotic Systems, Vol. 9, Paper 234, 10 pages, doi: $10.5772 / 53235$

[21] Švaco, M.; Šekoranja, B.; Jerbić, B. (2011). Autonomous planning framework for distributed multiagent robotic systems, Camarinha-Matos, L. M. (ed.), Technological Innovation for Sustainability, Springer, Berlin, 147-154, doi:10.1007/978-3-642-19170-1_16

[22] Švaco, M.; Šekoranja, B.; Jerbić, B. (2012). Industrial robotic system with adaptive control, Procedia Computer Science, Vol. 12, 164-169, doi:10.1016/j.procs.2012.09.048 